\title{
High levels of bilirubin as a risk factor for symptomatic gallstone disease
}

A large Danish study has shown that extreme levels of bilirubin in plasma are associated with an increased risk of developing symptomatic gallstone disease.

The researchers used a Mendelian randomization approach to test the association in 61,212 people from the general population of Denmark during a follow-up of 34 years. "Put simply, if one can show that the risk factor, bilirubin levels, associates with risk of gallstone disease; that there is a genetic variant or variants with a large effect on the level of the risk factor, bilirubin levels; and that the same genetic variant associates with risk of the clinical outcome, gallstone disease, it is likely that there is a causal relationship between risk factor and clinical outcome," explains author Anne Tybjærg-Hansen (Copenhagen University Hospital).

The authors found that participants in the tenth decile for plasma levels of bilirubin (which probably reflect biliary levels of bilirubin) had a greater risk of developing symptomatic gallstone disease than participants in the first to ninth deciles. They then tested whether a variant in the bilirubin glucoronidating gene (UGT1A1 rs6742078) was associated with increased plasma levels of bilirubin. Participants who were TT homozygous had much higher levels of bilirubin than participants who were GT heterozygous or GG homozygous. The effects were similar in men and women. TybjærgHansen and colleagues calculated that the UGT1A 1 genotype accounted for $20 \%$ of the variation in plasma levels of bilirubin.

These results indicate that "extreme bilirubin levels appear to be causally related to risk of gallstone disease," concludes Tybjærg-Hansen.

\section{Claire Greenhill}

Original article Stender, S. et al. Extreme bilirubin levels as a causal risk factor for symptomatic gallstone disease. JAMA Intern. Med. doi:10.1001/ jamainternmed.2013.6465 